Théologiques

Théologiques

\title{
Mutation technique et changement culturel
}

\section{André Gorz}

Volume 3, numéro 2, octobre 1995

Crise du travail, crise de civilisation

URI : https://id.erudit.org/iderudit/602425ar

DOI : https://doi.org/10.7202/602425ar

Aller au sommaire du numéro

\section{Éditeur(s)}

Faculté de théologie de l'Université de Montréal

\section{ISSN}

1188-7109 (imprimé)

1492-1413 (numérique)

Découvrir la revue

\section{Citer cet article}

Gorz, A. (1995). Mutation technique et changement culturel. Théologiques, 3(2), 72-88. https://doi.org/10.7202/602425ar

\section{Résumé de l'article}

Cet article s'inscrit d'abord en faux contre les « solutions » aux problèmes du chômage et de l'exclusion sociale, qui ignoreraient le caractère structurel de la crise du travail-emploi. La dynamique économique et technique fait maintenant disparaître les emplois à un rythme accéléré, mettant ainsi fin à la société salariale. Au lieu des politiques de «l'emploi pour l'emploi » et de la voie d'un " revenu d'existence », sources d'une nouvelle dualisation, il faut réaliser une mutation politique et culturelle qui déconnecte la continuité du revenu de la continuité de l'emploi et qui fasse reposer le lien social tant sur la prestation par tous d'un travail de moins en moins important que sur l'importance croissante reconnue à d'autres types d'activité. 


\section{Le travail n'est plus ce qu'il était}

Ce que nous appelons « travail " est une invention de la modernité. La forme sous laquelle nous le connaissons, pratiquons et plaçons au centre de la vie individuelle et sociale, a été inventée, puis généralisée avec l'industrialisme. Le «travail », au sens contemporain, ne se confond ni avec les besognes, répétées jour après jour, qui sont indispensables à l'entretien et à la reproduction de la vie de chacun; ni avec le labeur, si astreignant soit-il, qu'un individu accomplit pour réaliser une tâche dont lui-même ou les siens sont les destinataires et les bénéficiaires; ni avec ce que nous entreprenons de notre chef, sans compter notre temps et notre peine, dans un but qui n'a d'importance qu'à nos propres yeux et que nul ne pourrait réaliser à notre place.

(...) La caractéristique essentielle de ce travail-là - celui que nous « avons ", " cherchons », « offrons » - est d'être une activité dans la sphère publique, demandée, définie, reconnue utile par d'autres et, à ce titre, rémunérée par eux.

André GORZ,

Métamorphoses du travail. Quête du sens. Critique de la raison économique. Paris, Éd. Galilée, 1988, p. 25. 


\title{
Mutation technique et changement culturel ${ }^{1}$
}

\author{
André GORZ \\ philosophe \\ France
}

\section{RÉSUMÉ}

Cet article s'inscrit d'abord en faux contre les a solutions » aux problèmes du chômage et de l'exclusion sociale, qui ignoreraient le caractère structurel de la crise du travailemploi. La dynamique économique et technique fait maintenant disparâttre les emplois à un rythme accélété, mettant ainsi fin à la société salariale. Au lieu des politíques de * l'emploi pour l'emploi * et de la voie d'un "revenu d'existence ", sources d'une nouvelle dualisation, il faut réaliser une mutation politique et culturelle qui déconnecte la continuité du revenu de la continuité de l'emploi et qui fasse reposer le lien social tant sur la prestation par tous d'un travail de moins en moins important que sur l'importance croissante reconnue à d'autres types d'activité.

\section{La mutation technico-économique}

La mode pré-électorale est de concentrer les attentions sur l'exclusion et la misère des exclus. C'est plus rassurant que de se préoccuper des causes de l'exclusion et d'un chômage de masse qui ne cesse de s'étendre. Car les exclus, "c'est pas nous, c'est les autres". Ils sont tombés dans un gouffre si profond de solitude, de dénuement, de néant social que le moindre geste fait pour eux est déjà un grand cadeau. Pour leur rendre service, on va donc chercher, par pure bonté, à leur donner - et quoi donc? Mais du «travail », voyons, et à créer pour eux - quoi donc? Mais de

1 Cer article a été écrit pour la revue Théologiques et pour la revue Échange et Projets où il a paru dans le no 73, février 1995, p. 21-29. 
"l'emploi ", voyons, qui, bien entendu, devra être non qualifié et très peu payé. Et, par une dérive étonnante, on va prétendre que cette "solution » à l'exclusion est tout bonnement la solution du chômage.

Or s'attaquer à l'exclusion est une chose, s'attaquer au chômage et à ses causes en est une autre. L'exclusion est le résultat d'un chômage devenu irréversible à force de durer; elle demande un traitement spécifique. Mais l'insertion des exclus dans des emplois faits tout exprès pour eux ne réduira pas l'ampleur ni la durée du chômage des autres, ne leur évitera pas de tomber, à la longue, dans l'exclusion à leur tour, et surtout ne fournira pas des emplois "normaux " aux exclus après qu'ils auront été réinsérés, resocialisés, requalifiés par un travail. Bref, les mesures de réinsertion sont palliatives, non curatives ni préventives si elles ne vont pas de pair avec une politique qui s'attaque aux causes du chômage.

Cette politique ne peut consister à faire des chômeurs une "sousclasse " ( underclass ", comme disent les Anglais) reléguée dans un très grand nombre d'emplois de service à bas salaire, faible qualification et utilité souvent douteuse. Cette politique doit obligatoirement comporter la redistribution continuelle des emplois, même hautement qualifiés, sur le plus grand nombre possible de têtes, avec réduction continuelle et multiforme de la durée du travail. Car la cause du chômage n'est pas d'abord l'inaptitude ou le manque de qualification des chômeurs. En France, 25\% des chômeurs nouvellement inscrits en 1992 et 1993 ont fait au moins deux années d'études supérieures; $50 \%$ ont au moins le bac. Aux États-Unis, selon le US Department of Labor, "entre maintenant et l'an 2005,30\% des diplômés des universités iront grossir l'armée des chômeurs et des personnes sous-employées ${ }^{2}$ ». En Allemagne, continuellement cirée en exemple, $75 \%$ des jeunes diplômés des universités ne trouvent que des emplois non qualifiés. Que l'on ne dise pas que c'est faute d'expérience professionnelle : l'Allemagne compte environ un million de chômeurs P-2 et P-3, ainsi que 75000 chômeurs diplômés d'une école d'ingénieurs ou d'une faculté des sciences. Le chômage des personnes professionnellement qualifiées y a triplé en deux ans. $\mathrm{Si}$, en tenant compte de tous les paramètres, la France compte près de cinq millions de chômeurs, l'Allemagne - où la durée du travail diminue pourtant d'année en année - en compte 7,5 millions, dont 1,5 million en stage de formation ou en TUC et 1,5 million qui ont renoncé à chercher un emploi, faute d'espoir d'en trouver. 
Il est généralement admis que la reprise de la croissance n'améliorera pas substantiellement la situation. En raison des sureffectifs et des réserves de productivité accumulées durant la phase de récession, la reprise s'accompagne d'une accélération des gains de productivité. Selon les estimations étonnamment convergentes de deux grands groupes de consultants - le Boston Consulting Group et McKinsey 85 Co -25 millions d'emplois sur un total de 90 disparaîtraient aux États-Unis, 9 millions sur un total de 33 disparaîtraient en Allemagne si les méthodes et techniques les plus avancées étaient appliquées partout où elles sont applicables ${ }^{3}$. L'industrie allemande aurait actuellement un sureffectif de 2,5 millions de salariés et des réserves de productivité de 30 à $40 \%$. Dans les administrations et services, les réserves de productivité atteindraient jusqu’à $50 \%$.

Au risque de chômage s'ajoute le risque de précarisation, en raison de l'extension à la main-d'œuvre de la gestion "à flux tendus ». Les entreprises concentrent leur activité sur ce qu'elles sont le plus aptes à faire avec le maximum d'efficacité et externalisent le reste des activités sur des personnels payés à la vacation, dont les heures et le salaire varient de semaine en semaine. La vague des "externalisations" aura entraîné pour 1993 une contraction de $30 \%$ de la masse des salaires distribués par les entreprises privées américaines. Les 500 plus grandes n'emploient plus que $10 \%$ de salariés permanents et à temps complet ${ }^{4}$. Ce modèle de rationalisation s'étend rapidement en Europe. Il fait disparaître la "culture d'entreprise " et "l'identité au travail». C'est, selon la formule américaine, "the end of work as marriage *. L'actif type tend à devenir un nomade qui, quel que soit son niveau de formation, travaille par intermittence pour diverses entreprises et exerce divers métiers. Le développement du télétravail ne peut qu'accélérer et accentuer le déclin de l'entreprise en tant que lieu d'insertion, et du travail en tant que source d'identité personnelle.

\section{Le travail marginalisé}

La crise n'a donc pas pour raison la seule pénurie d'emplois; elle est due également aux changements dans la nature du rapport salarial. La société ne se divise pas simplement en "travailleurs " et "chômeurs",

3 Chiffres cités respectivement dans The Wall Street Journal, 19-20 mars 1993, et Heinrich HENZLER, directeur de McKinsey Allemagne, et Lothar SPÄTH dans Sind die Deutschen noch zu retten? Munich, Bertelsmann, 1993.

4 Voir « Disposable Workers » par Janice CASTRO, Time, 19 avril 1993. 
"inclus " et " exclus"; elle comprend une masse croissante, virtuellement majoritaire, de personnes qui ne sont "inclues" que de façon intermittente, précaire, partielle, toujours provisoire.

C'est pourquoi la société salariale est déjà dépassée dans les faits. La sauver, la reconstituer exigerait la création d'emplois permanents assez nombreux et assez consistants pour que le travail socialement rémunéré redevienne l'occupation principale, l'intérêt dominant et la source principale de l'identité sociale, professionnelle et personnelle de chacun. Le fait est qu'il ne peut plus être tout cela. La mort de la société salariale est aussi, plus fondamentalement, la mort de ce que Hannah Arendt appelait la "société de travailleurs". Celle-ci s'est défaite par suite de la précarité croissante du rapport salarial; de la disparition de la plupart des vrais métiers; du temps très limité que le travail-emploi occupe dans la vie de chacun; de l'inconsistance des tâches, trop abstraites, partielles, spécialisées ou fonctionnelles pour que la personne qui les remplit puisse être fière de ce qu'elle accomplit.

Si cette marginalisation du travail-emploi n'apparaît pas explicitement dans le discours social dominant, c'est avant tout que l'emploi - et surtout l'emploi stable, à temps complet - et les sécurités qu'il procure sont devenus des biens rares, convoités par celles ou ceux qui n'y ont pas accès et défendus jalousement. par leurs détenteurs qui, tous, doivent craindre d'en être dépossédés. D'où un double mouvement, en apparence contradictoire, de désaffection croissante vis-a-vis du travail et d'attachement croissant à l'emploi qu'on occupe ou convoite. Si, comme le soutiennent certains auteurs, «l'aspiration au temps libre n'est pas définitivement établie ", la raison n'en est pas que l'attachement et "l'identité au travail " resteraient forts mais - comme l'ont montré des enquêtes allemandes - que les conditions ne sont pas encore réunies qui permettraient aux actifs de combiner moins de travail-emploi et plus d'activités choisies, sans perdre les chances d'évolution professionnelle, la reconnaissance et les sécurités attachées à un emploi à temps plein.

Une politique de redistribution du travail et de création d'emplois d'intérêt collectif peut aboutir à ce que chacun et chacune puissent trouver une activité socialement reconnue, directement ou indirectement rémunérée; elle ne peut pas aboutir à ce que cette activité soit un emploi permanent et à plein temps occupant la majeure partie de la vie de chacun. Car

5 Voir Daniel Mothé et Jean-Louis LAVILLE dans Cohésion sociale et emploi. Paris, Desclée de Brouwer, 1994, p. 85 ss. et p. 115 ss. 
non seulement la majorité des emplois tendent à devenir intermittents ou à temps partiel; les activités non rentables au sens de l'économie classique ne pourront se développer et occuper le temps devenu disponible par la contraction du volume de travail, que si elles prennent une forme autre que celle du travail professionnel salarié.

Fondamentalement, le problème qui se pose aux sociétés post-industrielles est de savoir comment et à quoi elles entendent employer le temps rendu disponible par les économies massives de travail qui résultent des mutations techniques en cours. Actuellement, la femme passe une moyenne de $8 \%$ de son temps de vie éveillé à son travail-emploi, l'homme $14 \%$. Les * workaholics * parviennent à un maximum de $16 \%$. D'ici dix ans, le travail occupera $10 \%$ et $13 \%$ respectivement de la vie des unes et des autres ${ }^{6}$. Dans 20 à 25 ans, il n'occupera, selon Jacques Delors, que 40000 heures par vie, contre 70000 heures actuellement? , soit une moyenne de 1000 heures par an.

Le travail-emploi "n'est plus dominant, écrit Roger Sue, que dans la mesure où on s'efforce de nous faire croire qu'il l'est encore ${ }^{8}$ "; c'est-à-dire dans la mesure où "le discours social dominant " persiste à présenter le hors-travail comme le temps de l'oisiveté, de la consommation, du divertissement, de l'ennui, de la solitude. Ceux qui, contre "les utopies du temps libéré ", font valoir qu' "il n'y a de dignité que dans le travail ", ignorent que "travail", dans cet adage, ne signifie pas emploi mais "œuvre », c'est-à-dire création dont la valeur sera également reconnue par "l'ouvrier» et par les autres. Or très peu d'emplois sont dans ce cas. L'isolement, la pauvreté, l'inactivité et finalement l'exclusion qui accompagnent le chômage, masquent le fait que cette société ne manque pas simplement d'emplois : elle manque plus encore de possibilités d' « œuvrer ", c'est-à-dire d'activités assez consistantes pour qu'il vaille la peine de s'y donner pleinement.

La très grande majorité cherche (souvent sans les trouver) ces activités dans le temps hors travail, ce qui conduit Roger Sue à dire que "le travail

6 «Un mondo fuori dal lavoro», entretien avec Claus OFFE. Il Manifesto, 29 juin 1993. Auteur de plusieurs ouvrages sur la crise de l'État-providence, C. Offe enseigne au Zentrum für Sozialpolitik de l'Université de Brême.

$7 \quad$ Chiffres cités par Jacques Delors dans L'unité d'un homme. Odile Jacob, 1994, et lors d'un exposé à l'IEP, le 3 novembre 1994.

8 Roger SUE, Temps et ordre social. Paris, PUF, 1994, p. 250. 
n'est plus le travail ${ }^{9}$ ". La solution à la désintégration de la société salariale est donc à chercher dans la transformation du temps devenant disponible en temps d'activités privées aussi bien que publiques, autrement dit : dans l'appropriation individuelle et sociale du temps liberé. Ce temps déjà dominant quantitativement doit devenir le temps dominant de la production de soi et de la production de société. Cette "inversion des temps sociaux ", comme l'appelle Joffre Dumazedier ${ }^{10}$ suppose évidemment une "inversion des valeurs " (Roger Sue) et une transformation des rapports sociaux; autrement dit, une mutation culturelle et politique :

1) culturellement, elle requiert une capacité d'autodétermination personnelle et d'auto-organisation sociale que la généralisation du rapport salarial, la spécialisation fonctionnelle du travail et l'extension des rapports marchands ont méthodiquement refoulée;

2) politiquement, elle demande que les activités développées hors de la sphère du travail-emploi et des échanges monétaires soient socialement légitimées par un statut qui leur confère la visibilité sociale et leur permette de s'inscrire dans l'espace public.

\section{L'inversion des valeurs}

1) Pour ce qui est du premier point, tout s'oppose, présentement, au développement des auto-activités : la structuration sociale de l'espace et du temps; la séparation des lieux de travail, de consommation et d'habitation; l'absence ou la rareté, dans les quartiers, d'ateliers pour les autoproductions et la création; d'espaces pour les rencontres, les échanges, les activités auto-organisées et, par conséquent, la difficulté à construire des réseaux de coopération, d'aide mutuelle, d'échange de savoirs et de services; enfin une conception de l'éducation qui - à la différence des pédagogies dont, de Rousseau à Klauss Haefner ${ }^{11}$, en passant par Maria Montessori, P.P. Blonski ${ }^{12}$ et Paul Oestereich ${ }^{13}$, le but est le plein

Roger SUE, op. cit.

10 Joffre DUMAZEDIER, La révolution culturelle du temps libre - 1968-1988. Paris, Méridiens Klincksieck, 1988.

11 Klaus HAEFNER, Die neue Bildungskrisse. Bâle, Birkhäuser, 1982 et Mensch und Computer im Jahr 2000. Bâle, Birkhäuser, 1984. Professeur d'informatique appliquée à l'enseignement, K. HAEFNER répertorie les capacités et activités « substiruables " par l'informatisation et insiste sur la nécessité de repenser l'éducation en privilégiant les capacités non substituables, intuitives, esthétiques, créatrices.

12 Pavel PAVLOVITCH BLONSKI, pédagogue russe (1884-1933), liait l'acquisition de connaissances à des activités pratiques qui en faisaient naître le besoin, à l'occasion 
développement de toutes les facultés, sensorielles, manuelles, intellectuelles et morales de la personne - privilégie outrancièrement l'hémisphère gauche du cerveau et néglige l'apprentissage des multiples savoirfaire dont dépend la richesse d'une culture "vernaculaire ", la capacité de faire face aux situations de la vie de tous les jours et de comprendre les rapports des hommes avec leur environnement.

Il n'est pas étonnant, dans ces conditions, que, selon le discours social dominant, "les gens ne savent que faire d'eux-mêmes quand ils ne sont pas au travail ", "les gens ne sont pas mûrs pour un mode de vie où l'emploi ne tiendrait qu'une place très réduite ». En réalité, il s'agit là avant tout de ce que Roger Sue appelle "une opinion sur l'opinion". "Aucune enquête, depuis dix ans, ne fait apparaître le travail au premier rang des valeurs les plus importantes. " "Jamais la réussite professionnelle n'est citée comme le facteur le plus important de la réalisation de soi. " $9 \%$ seulement des personnes interrogées ( $7 \%$ des jeunes de 18 à 25 ans) citent le travail comme le "principal moyen de réussir sa vie". L'inversion des valeurs s'est accomplie sans pour autant se refléter dans le discours social et politique; le temps hors travail est pourtant déjà vécu comme le temps dominant de la production de soi et de la production de sens, même chez ceux, notait Alain Lebaube à propos d'une enquête auprès de jeunes diplômés des grandes Écoles, qui ont été "sélectionnés $a$ priori pour épouser la cause de l'efficacité et de la motivation ${ }^{14}$ ». "Le temps libre impose ses valeurs au temps dominé ", "le choix de l'emploi est fonction du temps qu'il laisse ${ }^{15}$ ". "L'identité " est une "patchwork identity $\gg$, selon l'expression de sociologues anglo-saxons, que chacun a à créer à partir des éléments disparates d'un monde fragmenté, en tâchant de "composer sa vie comme on compose une cuvre d'art ${ }^{16}$ ». Faute d'identité et de place prédéterminée dans une société en continuel changement, chacun a à se produire soi-même à partir d'appartenances mul-

notamment des " robinsonnades d'été " et, l'hiver, de missions d'exploration et d'observation des activités urbaines.

13 Paul OESTEREICH, pédagogue allemand (1878-1957), donnait pour mission à l'école d'épanouir la totalité de la personne en : a) familiarisant avec la vie de tous les jours; et b) développant l'autonomie intellectuelle et morale. Acquisition de connaissances et de savoir-faire, savoir-être, savoir-vivre sont intimement liés.

Voir Le Monde - Initiatives, 22 janvier 1992.

15 Roger SUE, op. cit., p. 210 et 223.

16 Alain TouraINe, Critique de la modemité. Paris, Fayard, 1992. 
tiples et mouvantes à des "bandes", des "cliques ", des clubs, des associations; par la danse, le sport, l'habillement, l'amitié, l'amour, beaucoup plus que par le travail, sans achèvement possible. C'est sur cette quête sans fin, dans laquelle affleure ou même s'affirme l'autonomie du sujet, que peut faire fond une politique qui reconnaît la valeur des activités autodéterminées.

\section{Le «droit au travail »}

2) Présentement, la société ne reconnaît que les activités dont "l'utilité sociale " donne lieu à une rémunération et à un statut. La notion d' « utilité sociale " remonte elle-même, comme le note Alain Touraine ${ }^{17}$, à l'époque où la société était un ordre que chacun avait à servir à la place qui lui était assignée par la naissance, la tradition familiale, le métier hérité des parents. On était "socialement utile " quand on tenait sa place et contribuait ainsi au fonctionnement et à la reproduction du corps social.

L'ordre social s'est dissous entre-temps. La société est devenue un système d'appareils et de flux qui, loin d'assigner une place à chacun, exige de tous la capacité de s'adapter aux changements continuels. L'idée d'utilité sociale en devient aussi caduque que celle d'intégration. Le système sélectionne plus qu'il n'intègre ceux qui doivent le servir. Le servir signifie accomplir de façon efficace et flexible le travail fonctionnellement spécialisé qu'exige la marche d'un ensemble d'appareils (industriels, administratifs, de communication, etc.). Le travail exigé doit concourir de façon prédéterminée à la réalisation d'un but lui-même prédéterminé et qui ne peut ni ne doit dépendre du jugement personnel de chacun. Les "travailleurs" sont en quelque sorte les fonctionnaires d'une machinerie qu'ils ont à servir $-y$ compris par leur jugement et des initiatives fonctionnellement circonscrits (ce que j'ai appelé l'autonomie au sein de l'hétéronomie) - mais dont ils n'ont à apprécier ni les finalités ni la conception.

L'intégration qu'assure le travail-emploi est donc, selon la distinction de Durkheim, fonctionnelle beaucoup plus que sociale, et il en est de même de l'utilité. Le travail procure au travailleur le sentiment d'être utile non pas à quelqu'un ou à quelque chose mais utile en soi, sans autre précision, de façon impersonnelle et anonyme à un tout social au fonctionnement abstrait, et donc investi de droits économiques et sociaux sur celuici. Ces droits ne sont pas attachés à sa personne mais à la fonction imper- 
sonnelle qu'il remplit dans le processus social de production: ils sont attachés à son emploi, quel que soit d'ailleurs le contenu de celui-ci ${ }^{18}$.

Le travail-emploi est seul présentement à ouvrir les droits économiques et sociaux constitutifs de ce que j'ai appelé la citoyenneté économique. Celle-ci comporte d'importants pouvoirs : notamment le pouvoir de contractation, de négociation et d'action collectives, d'organisation syndicale. Elle apporte aux actifs - ou leur apportait avant la vague de précarisation, flexibilisation, déréglementation - une sécurité matérielle et morale : le travailleur est reconnu utile a la société independamment de son intention d'etre tel. Il n'a pas à répondre ni à se préoccuper de l'utilité ni de la moralité de ce qu'il fait; celle-ci est postulée inhérente au fait d'être employé.

Le travail-emploi a ainsi pour les individus une valeur (au sens moral et social) indépendante de la valeur du travail auquel ils sont employés. Aussi, la grande majorité des gens aiment avoir un emploi sans aimer l'emploi qu'ils ont. Celui-ci vaut par sa nature d'emploi, non dans sa nature de travail, en raison des droits économiques et sociaux qu'il conferre. Dès lors cependant qu'il est source de droits, source de citoyenneté, le travail-emploi doit apparaître comme étant lui-même un droit qui, au nom de l'égalité des droits de tous les citoyens, doit être accessible à tous. Le principe d'utilité au nom duquel les droits économiques et sociaux ont été attachés au travail est ainsi transcendé par la normativité juridique attachée à ces droits. Aucune considération économique ou technique n'est

18

L'idée que dans la « société de service » qui serait la nôtre, la grande majorité des emplois correspondent à la demande de personnes concrètes, auxquelles les salariés doivent vendre leurs services et apprendre à se vendre (Bernard Perret), relève de l'idéologie beaucoup plus que de l'observation. Elle vaut pour les seuls services dont la consommation doit être induite par l'offre et la séduction du consommareur par le vendeur. Or ces services de personne à personne sont loin d'être ceux qui emploient le plus de personnel. Dans les banques et assurances, postes et télécommunications, transports, seule une faible minorité des personnels est en contact avec la clientèle. Dans le commerce de détail et la restauration - qui, ensemble, fournissent aux ÉtatsUnis $45 \%$ de tous les emplois -, l'importance des personnels de manutention, entretien, administration, préparation, etc. dépasse de loin celle des vendeurs et serveurs. Les premiers, d'autre part, ne sont pas d'abord des personnes au service des désirs d'autres personnes mais les serviteurs d'entreprises dont le but est de vendre tout ce qui - utile ou inutile, bon ou mauvais - peut être vendu, la séduction du client, la production de la demande étant beaucoup plus le fait de la publicité (rebaptisée acommunication») . dont les professionnels ne sont pas en contact direct avec les personnes - que du personnel de vente. 
opposable au droit de tous d'accéder à la citoyenneté économique. Le droit au travail - expression qui n'a de sens que si "travail " y est synonyme d'emploi - est donc perçu comme un droit politique. Sa violation est inacceptable puisqu'elle crée deux catégories de citoyens. La tolérer revient à accepter que le pouvoir des "décideurs économiques "l'emporte sur le droit républicain (et sur la Charte des Droits de l'Homme).

\section{Au-delà de l'utilité sociale}

Dans un contexte où la société a des besoins de plus en plus faibles de travail, le droit à la citoyenneté économique tend inévitablement à être dissocié du critère de l'utilité sociale. Cette dissociation peut prendre différentes formes:

a) La première, qui est encore la plus répandue, consiste à vouloir multiplier les emplois en tant que supports de la citoyenneté économique, indépendamment de leur utilité ou inutilité. On pense ainsi à multiplier les emplois de guichetier, caissier, pompiste, laveur de pare-brise, portier, hôtesse, coursier, etc.; à inciter les entreprises non exposées directement à la concurrence étrangère à utiliser par unité de produit plus de travail que nécessaire; ou encore à subventionner fiscalement les emplois de personnel de maison dans les ménages même valides et aisés.

b) La seconde forme de dissociation de la citoyenneté économique est l'institution d'un "revenu d'existence " garanti indépendamment de toute activité. Cette formule ne conferre toutefois pas l'intégralité des droits économiques et sociaux attachés au travail-emploi : octroyé par voie législative, le revenu d'existence ne peut être négocié, ne confère pas le pouvoir de contractation, d'action collective et d'organisation syndicale. Son montant étant le même pour tous, quel que soit le niveau des autres revenus, les personnes qui dépendent le plus du revenu d'existence pour leur subsistance ne peuvent mener des actions organisées spécifiques pour faite valoir leurs besoins.

c) La troisième forme de dissociation entre citoyenneté économique et utilité sociale consiste à ouvrir beaucoup plus largement l'eventail des activités qui méritent d'être socialement reconnues. Cette extension des critères de reconnaissance sociale prend tout son sens dans un contexte où le travail discontinu, intermittent, à temps très réduit tend à devenir la règle et où la norme d'un "plein temps" devient elle-même très infé- 
rieure à ce qu'elle est présentement ${ }^{19}$. Quand le travail-emploi cesse d'être le principal "lien social ", l'individu ne peut plus se définir par sa manière de servir la société qui le produit, ni la société par sa manière de se servir des individus pour sa reproduction. L'individu est désormais en situation d'avoir à produire de la société en se produisant lui-même et inversement. Au rapport unilatéral d'utilité doit dès lors succéder - et tend déjà à succéder — un rapport dans lequel la société reconnaît la valeur du sujet en tant qu'il s'auto-produit en produisant de la société. Le souci de l'épanouissement de la personne l'emporte alors sur le souci d'ascension dans la hiérarchie sociale et de conformité. "La societé devient un moyen de l'épanouissement de l'individu", écrit Christian Lalive d'Epinay 20 .

Ce changement de paradigme trouve son expression dans le changement culturel (le "Wertewandel * des Allemands) dont le mouvement des femmes, le mouvement écologiste, le souci de la qualité de la vie et de l'environnement sont les formes les plus visibles. La raison instrumentale, c'est-à-dire l'utilisation des choses et des gens comme moyens en vue de fins qui elles-mêmes sont des moyens en vue d'autres fins, et ainsi de suite, est contestée au nom d'une éthique du souci de soi, des choses, des autres, de tout ce qui est fragile et demande à être protégé et soigné : ce que les Anglais appellent * ethics of care * ou * caring *, les Allemands le nomme la prééminence des "valeurs féminines". Ainsi, dans la charte du SPD adoptée en 1989, on lit: "Les hommes aussi prennent conscience de ce que la subordination prétendument virile du sentiment et de l'imagination à la rationalité et à l'efficacité les appauvrit ou même les rend malades. Pour que la société devienne humaine, il faut qu'elle cesse d'être virile" et, en libérant le temps, donne de l'espace à "l'amour et à la convivialité, au rêve et au retour sur soi ${ }^{21}$ ». De même, Anthony Giddens, dans un long article sur l'avenir du parti travailliste britannique, propose de définir le "socialisme " par la primauté donnée aux activités non instrumentales consistant à "prendre soin " (care).

19 L'International Herald Tribune du 27 octobre 1994 rapporte une déclaration d'Akio Morita préconisant la semaine de 30 heures et l'allongernent des congés payés, en faisant valoir que la qualité et la capacité d'évolution d'une main-d'œuvre dépendent de la possibilité qui lui est donnée de prendre du champ et de «changer de paysage * (Akio Morita est le fondateur et PDG de Sony).

20 Voir la dernière référence de ce texte, p. 88.

21 Une analyse de certe Charte, inédite en France, se trouve dans A. GORZ, Capitalisme, socialisme, écologie. Paris, Galilée, 1991, chapitre 4. 
Prendre soin implique une éthique de la responsabilité de soi, des autres et de la texture du monde matériel. En prendre soin est le contraire de l'égoïsme mais ne doit pas être confondu avec l'altruisme. Car le souci de soi - l'attitude responsable envers soi-même et son corps - est à la source de la capacité de se soucier des autres 22 .

Une politique de réappropriation individuelle et collective des plages de temps, soustraites au travail grâce aux mutations technico-économiques, doit donc se comprendre avant tout comme l'extension du pouvoir des individus sur leur propre vie et comme l'émancipation de la société des contraintes systémiques dans sa production d'elle-même. La flexibilité et la discontinuité croissantes du rapport salarial, la diminution du volume de travail nécessaire à l'économie peuvent, dans cette perspective, être socialement transmutées en sources de nouvelles libertés : en l'extension des droits sur eux-mêmes que, indépendamment de tout critère d'utilité, la société moderne reconnaît aux individus.

\section{La réappropriation du temps}

Tel est précisément le sens d'une "politique du temps" 23 ". Elle a pour objet de permettre à tous et à toutes de gagner leur vie en travaillant de façon continue ou discontinue, à temps partiel ou à temps complet et de pratiquer toutes les formes d'alternance et de combinaison entre travail hétérodéterminé et activités autodéterminées, toutes les formes de "temps choisi " (à l'échelle de la journée, de la semaine, du mois, de l'année, etc.) dont la possibilité est déjà inscrite dans les formes d'emploi temporaire, intermittent, à durée flexible, à horaires variables. Aucune de ces formes de flexibilité n'a de raison de relever du pouvoir régalien de l'employeur. Toutes peuvent faire l'objet d'une réappropriation collective et individuelle par les salariés. La flexibilité négociée peut et doit prendre la place de la flexibilité imposée. Elle peut être poussée jusqu'à la suppression de toute contrainte horaire 24 , jusqu'au libre choix par chacun de

22 Anthony GIDDENS, “What's Left for Labour „, New Statesman and Society, 30 septembre, 7 et 14 octobre 1994.

23 Voir "Échange et Projers *, La révolution du temps choisi. Paris, Albin Michel, 1980 , qui reste une référence indépassable dans ce domaine.

24 La fabrique de moteurs Landert à Oerlikon (Zurich) s'est rendue célèbre en organisant le travail de manière à laisser à chacun une totale liberté dans le choix de ses horaires. La même liberté a été obtenue par les fonctionnaires du Québec et une partie du personnel administratif de Siemens. 
sa durée du travail et de ses horaires ${ }^{25}$. L'emploi intermittent lui-même peut être transformé en droit de toute personne de travailler de façon discontinue, même lorsqu'elle occupe un emploi stable, à temps complet et horaires réguliers. Ce droit revient en fait à assimiler à des périodes de travail des périodes d'activité non rémunerée durant lesquelles continuera d'être versé un revenu proche du salaire normal de la profession de chacun. Ce droit, relativement développé en Suède, y permet aux parents de se partager à leur guise, durant les trois années qui suivent la naissance d'un enfant, douze mois de congé parental d'éducation avec $90 \%$ de leur salaire respectif.

En fait, le droit au travail discontinu avec revenu continu, ainsi que le travail à durée variable avec un revenu qui ne varie guère existent déjà sans oser dire leur nom; ils existent sous la forme, entre autres, de l'indemnisation du chômage total ou partiel. Cette indemnisation est de 80 à $90 \%$ du salaire normal dans plusieurs pays de l'Union Européenne. Au Danemark, tout salarié peut céder son emploi à un chômeur pour un an et toucher pendant cette année plus de $70 \%$ de son salaire normal. D'autres systèmes de partage du travail par réduction de sa durée, sans réduction proportionnelle du salaire, sont pratiqués ${ }^{26}$ et envisagés, ainsi que différents systèmes de revenu garanti pendant les interruptions du rapport salarial. Seules toutefois, j'y insiste de nouveau, confèrent les pouvoirs attachés à la citoyenneté économique les garanties de revenu rattachées à un revenu négociable et qui tombent elles-mêmes, de ce fait, dans le champ de la négociation collective.

La notion d' "indemnité de chômage " sera devenue définitivement obsolète quand «le travail à temps partiel deviendra la règle pour l'organisation d'une vie, en raison de la diminution du temps consacré au

25 Aux grands magasins Beck à Munich, chaque employé peut choisir au début de chaque mois son nombre d'heures de travail sans que sa paie varie. Une durée mensuelle moyenne de travail est choisie par chacun en début d'année et les employés peuvent prendre de l'avance ou du retard sur cette moyenne à l'échelle du mois, à condition de réaliser la moyenne en fin d'année. La coordination er l'arbiurage entre les choix individuels ont lieu au début de chaque mois en assemblée générale du personnel de chaque service.

26 Dont le « dix pour un » proposé récemment par Nikonoff et que pratique le syndicat des transports publics de Copenhague : neuf employés font embaucher un chômeur et réduisent la durée du travail de chacun en conséquence. La perte de salaire est partiellement indemnisée. Ce système n'est évidemment applicable que pour des emplois permanents à horaires réguliers. 
travail contraint 27 ". La différence entre temps complet et temps partiel, emploi intermittent ou permanent s'estompera alors. Chaque emploi ou poste sera tenu par deux ou plusieurs personnes travaillant alternativement à temps plein ou simultanément à temps réduit, selon leur convenance. Cette généralisation du temps partiel, au lieu de signifier comme c'est actuellement le cas dans le monde anglo-saxon - la généralisation du chômage et du salaire partiels, signifiera la nécessaire généralisation d'un revenu binôme, dont la composante sociale, indexée sur le salaire conventionnel, remplace l'indemnité de chômage 28 . On peut imaginer que pour avoir droit au versement ininterrompu de ce revenu social, proche du salaire normal d'activité, chacun devra accomplir un volume minimum de travail ou d'activité assimilée au travail. La plus grande liberté dans l'organisation par chacun de sa vie sera permise par un volume de travail défini à l'échelle non pas d'une mais de trois ou quatre années par exemple.

Si on se place dans cette perspective de généralisation du travail à temps partiel, on ne peut en même temps vouloir transformer en emplois toutes les activités que les personnes peuvent assumer individuellement ou collectivement dans leur temps disponible. Prospecter systématiquement les "gisements d'emploi ", ne considérer les "services de proximité " que comme un de ces "gisements ", c'est postuler qu'il ne peut y avoir d'autre lien social que le travail-emploi et, par une potitique de "l'emploi pour l'emploi ", introduire une nouvelle coupure dans la société : d'un côté l'élite du travail, spécialisée dans les activités fonctionnelles à productivité économique élevée; de l'autre côté les professionnels des activités plus relationnelles et conviviales, dont l'élite du travail serait dispensée. "Spécialistes sans âme, jouisseurs sans cœur », disait Max Weber.

Cette nouvelle dualisation ne peut être évitée que si on se place d'emblée dans la perspective d'une participation de chacun aux deux secteurs d'activité : celui du travail performant, économiquement nécessaire, et celui des activités relationnelles et conviviales. Il faut alors voir les "services de proximité " "non comme une solution au chômage mais comme une solution au temps libre 29 . Certes, ces services ne peuvent se développer et se perpétuer par la seule auto-organisation spontanée de réseaux d'aide mutuelle. Ils ont besoin du soutien d'une structure stable

Jacques DELORS, op. cit., p. 331.

28 C'est l'idée de généralisation du « deuxième chèque », lancée par Guy AZNAR. Voir notamment Travailler moins pout travailler tous. Paris, Syros, 1994.

29 Roger SUE, Vers une sociétê du temps libre? Paris, PUF, 1982, p. 164. 
qui assure leur continuité de fonctionnement et forme des bénévoles à l'accomplissement de tâches qui demandent une qualification plus poussée. Mais ce soutien par des services sociaux publics et des prestataires de service privés ne doit être que subsidiaire. Il fournira des emplois en nombre limité, car tel n'est pas son but. Son but est de favoriser le développement de liens sociaux vécus de solidarité et d'entraide.

Ce qui est en question, écrit Bernard Ginisty, c'est tout simplement l'art de vivre. Vieux ou jeunes, malades ou bien portants, nous avons tous besoin de services professionnels compétents. Mais nous entendons rester les sujets de notre existence et non démissionner entre les mains de professionnels "supposés savoir ". Il est urgent de créer des espaces micro-sociaux qui ne se réduisent pas à être clients d'administrations, de professionnels ou d'hommes politiques ${ }^{30}$.

\section{En attendant}

Des mesures spécifiques pour réinsérer les exclus et prévenir l'exclusion restent nécessaires en attendant que soit mise en œuvre et que porte ses fruits une politique de redistribution du travail par la réduction continuelle, périodique, multiforme de sa durée. Sur les modalités et le financement d'une telle politique, Guy Aznar et moi nous sommes étendus ailleurs.

11 faut prendre garde, en attendant, que les mesures spécifiques à l'intention des exclus et des personnes menacées d'exclusion ne colonisent par avance des espaces dans lesquels pourront se déployer les activités motivés par le " caring * et non par "l'utilité sociale ". L'une des voies qui s'offre est la création d'une sorte de service civil prolongeable qui offre le choix entre un large éventail d'activités à forte visibilité et valeur sociale.

Dans le modèle danois de Frederickshavn, ces activités sont rémunérées par un revenu suffisant pour vivre normalement et adaptées aux capacités et aspirations des personnes. Elles peuvent aller de travaux d'entretien des espaces publics et de l'environnement jusqu'aux cours d'art dramatique ou la formation d'un orchestre.

Le modèle genevois garantit " un minimum social permettant de couvrir les besoins des bénéficiaires ». Ce minimum garanti est de 13.812 francs suisses par an pour une personne, ou 20.162 francs pour un couple

30 Bernard GiNiSTY, « Je ne veux pas devenir un gisement d'emploi », Bulletin du RFAS, 1992. Dans le même esprit, voir Roger SUE, op. cit., p. 157-167. 
(soit respectivement $15672 \$$ et $22877 \$$ ), à quoi viennent s'ajouter « le loyer, l'assurance maladie de base ainsi que certains frais de vêtement et de maladie" .

Avec ce minimum garanti aux chômeurs en fin de droits et aux jeunes ne trouvant pas de travail, la loi "introduit une notion nouvelle qui est la contre-prestation d'utilité sociale ou environnementale dont le temps consacré à cette activité sera nettement inférieur à celui d'une activité professionnelle. Cette contre-prestation doit essentiellement être considérée comme un lien, un contrat qui lie le bénéficiaire et la société. Elle doit être considérée comme un élément revalorisant et non pas comme une contrainte. Elle ne doit pas être la création de "petits boulots" précaires... »

" Un certain nombre de lieux potentiels ont déjà été définis dans les établissements de soins et d'éducation, les lieux socio-culturels, les associations d'action sociale subventionnées, les lieux municipaux et cantonaux ouverts au public, restaurants scolaires, musées, bibliothèques, centres sportifs, archives de l'État, structures de prise en charge des personnes âgées, etc. La formation et le recyclage restent prioritaires."

L'un des principaux inspirateurs de cette loi, Christian Lalive d'Epinay, en résume l'esprit dans des * thèses prospectives sur le XXI siecle », notamment celles-ci :

La société devient un moyen au service de l'épanouissement de l'individu. Le lien social de la société post-industrielle ne saurait en aucun cas être fondé sur le facteur travail-emploi. L'épanouissement de l'homme peut parfaitement être réalisé non seulement par une activité privilégiée mais par une gamme d'activités allant du loisir à l'activité rémunérée en passant par un engagement volontaire au service de la collectivité. En conséquence la norme n'est plus l'aptitude au travailemploi mais l'aptitude à la vie autonome et cela à l'intérieur de contraintes précises, le revenu et l'état de santé étant les plus lourdes. Cela nous amène à envisager des formules d'échanges entre l'individu et la société (...) selon lesquelles le revenu garantissant le droit individuel à l'existence serait, partiellement en tout cas, déconnecté de l'emploi. Cette approche favoriserait l'émergence d'une société où l'individualisme n'exclurait pas la solidarité (...) et où la sortie du marché du travail ne serait pas une marginalisation ${ }^{31}$. 